Review

\title{
Flavonoids and Melanins: A Common Strategy across Two Kingdoms
}

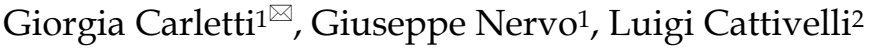 \\ 1. Consiglio per la Ricerca e la Sperimentazione in Agricoltura, Research Unit for Intensive Wood Production, Strada Frassineto 35 , 15033 Casale \\ Monferrato, AL, Italy; \\ 2. Consiglio per la Ricerca e la Sperimentazione in Agricoltura, Genomics Research Centre, via S Protaso 302, I-29107 Fiorenzuola d'Arda, PC, Italy.
}

$\triangle$ Corresponding author: Tel: phone +39 0142 330900; E-mail:giorgia.carletti19@gmail.com.

( ) Ivyspring International Publisher. This is an open-access article distributed under the terms of the Creative Commons License (http://creativecommons.org/ licenses/by-nc-nd/3.0/). Reproduction is permitted for personal, noncommercial use, provided that the article is in whole, unmodified, and properly cited.

Received: 2014.05.16; Accepted: 2014.10.08; Published: 2014.10.29

\begin{abstract}
Ultraviolet (UV) radiations alter a number of metabolic functions in vivant. They produce damages to lipids, nucleic acids and proteins, generating reactive oxygen species such as singlet oxygen $\left(\mathrm{O}_{2}\right)$, hydroxyl radical $(\mathrm{HO})$ and superoxide anion $\left(\mathrm{O}_{2}{ }^{-}\right)$. Plants and animals, after their water emersion, have developed biochemical mechanisms to protect themselves from that environmental threat through a common strategy. Melanins in animals and flavonoids in plants are antioxidant pigments acting as free radical scavenging mechanisms. Both are phenol compounds constitutively synthesized and enhanced after exposure to UV rays, often conferring a red-brown-dark tissue pigmentation.

Noteworthy, beside anti-oxidant scavenging activity, melanins and flavonoids have acquired secondary functions that, both in plants and animals, concern reproductions and fitness. Plants highly pigmented are more resistant to biotic and abiotic stresses. Darker wild vertebrates are generally more aggressive, sexually active and resistant to stress than lighter individuals. Flavonoids have been associated with signal attraction between flowers and insects and with plant-plant interaction. Melanin pigmentation has been proposed as trait in bird communication, acting as honest signals of quality.

This review shows how the molecular mechanisms leading to tissue pigmentation have many functional analogies between plants and animals and how their origin lies in simpler organisms such as Cyanobacteria. Comparative studies between plant and animal kingdoms can reveal new insight of the antioxidant strategies in vivant.
\end{abstract}

Key words: antioxidant strategy, pigments, horizontal gene transfer (HGT); plants, animals, metabolism, genetic bases, mutants.

\section{Introduction}

Ultraviolet (UV) rays are electromagnetic radiations with a wavelength from 10 to $400 \mathrm{~nm}$. The UV radiations are produced by the sun and part of them, those between 290 and 400nm, reach the Earth's surface. UV-C (from 280 to $100 \mathrm{~nm}$ ) and short UV-B (from 315 to $280 \mathrm{~nm}$ ) are strongly harmful for vivant life and only the presence of the ozone layer has allowed the development of the life on Earth. Before the formation of the ozone layer, the Earth's surface was subjected to a high level of UV radiations and the life was permitted in aquatic environments only, since water acts as UV filter [1].

UV are the major exogenous source of Reactive Oxygen Species (ROS) such as hydrogen peroxide $\left(\mathrm{H}_{2} \mathrm{O}_{2}\right)$, superoxide radical $\left(\mathrm{O}_{2}{ }^{-}\right)$, hydroperoxyl $\left(\mathrm{HO}_{2}{ }^{-}\right)$ and singlet oxygen $\left({ }^{1} \mathrm{O}_{2}\right)$ [2]. The occurrence of one unpaired electron results in high reactivity of these species due to their affinity to donate or receive an 
electron to attain stability. The balance between the presence of both pro-oxidants and antioxidants is tightly regulated and extremely important for maintaining vital cellular and biochemical functions. Changing the balance towards an increase in the pro-oxidant over the capacity of the antioxidant is defined as oxidative stress.

UV-B can produce either direct or indirect damages to living organisms. The absorption of photons of UV-B radiations can directly damage the DNA molecules breaking their chemical bonds. The unbounded base interacts with adjacent bases on the same DNA strand to create new bonds and forming dimmers between two adjacent pyrimidines (cytosine and/or thymine) on the same DNA strand. The two major DNA lesions induced by UV-B radiation are CPDs (cyclobutane pyrimidine dimers) and 6-4PPs (pyrimidine (6-4) pyrimidone photoproducts). Occasionally, the lesions within the DNA interfere with the proper translation of a gene, altering or impairing its function. Accumulation of mutations in key genes due to chronic exposure to UV can lead to the development of skin cancer [3]. Beside DNA damage, exposure to UV radiations can also produce indirect effects, leading to lipid peroxidation (oxidations of polyunsaturated fatty acids), oxidations of amino acids, as well as oxidative inactivation of specific enzymes by oxidation of their co-factors [4].

Considering the risks associated with UV exposure, the development of UV protective mechanisms has been a priority for the evolution of all organisms. To allow the water emersion and to protect themselves from that environmental threat, the living organisms have evolved a number of pigments with sunscreen capacity and/or antioxidant activity. Noteworthy, all organisms under the same evolutionary pressure had evolved very similar protective molecules. In this review we explain how plants and animals, although they evolved independently, share common photo-protective mechanisms. Plants and animals produce two classes of pigments (flavonoids and phenylpropanoids in general in plants, and melanins in animals) characterized by sunscreen capacity and free radical-scavenging properties and, consequently, able to filter UV radiations and to neutralize the ROS, originated during oxidative stress. Both classes of molecules beside their UV-protection activity are also implicated in other biological functions playing important roles in the interaction with the environment (e.g., protection from biotic and abiotic stresses) and in the interaction among individuals (e.g., communication and reproduction).

\section{Pigments as Sunscreen Molecules}

Epidermal melanin pigmentation has been con- sidered as a primary absorber of UV radiation thus providing protection to the underlying epidermal and dermal elements. The shielding effect of melanin, especially eumelanin, is achieved by its ability to serve as a physical barrier that scatters UV radiations, and as an absorbent filter that reduces the penetration of UV through the epidermis (Kaidbey et al., 1979) [5].

Melanin in black skin is twice as effective compared to white skin in inhibiting the penetration of UV-B radiations. Black epidermis allow only $7.4 \%$ of UVB and $17.5 \%$ of UVA to penetrate, $24 \%$ UVB and $55 \%$ UVA passes through white skins [6]. Further, melanosomes in dark skin are resistant to degradation by lysosomal enzymes, they remain intact throughout the epidermal layers [7] and they form supranuclear caps in keratinocytes and melanocytes which contribute considerably to photoprotection against UV-induced damage [8]. In contrast, in lightly pigmented skin, melanosomes are degraded and only persist as "melanin dust" in the suprabasal layers. This reduction of melanosomes in the upper epidermis is considered an important factor in carcinogenesis, as it compromises the photoprotection of the skin [9].

In higher plants, flavonoids and other phenylpropanoid derivatives (such as sinapate esters) that accumulate in large quantities in the vacuoles of epidermal cells, effectively attenuate the UV component of sunlight with minimal effects on the visible region of the spectrum. In field-grown soybean, significant differences have been revealed in UV penetration among cultivars with different levels of leaf phenolics, and between plants grown under contrasting levels of solar UV-B [10]. Arabidopsis mutants compromised in their ability to produce flavonoid derivatives (transparent testa5, tt5) or sinapate esters (ferulicacidhydroxylase1, fah1) were more susceptible to UV-B than wild-type. Enhanced penetration of UV-B through the epidermis of sunscreen-deficient plants leads to an increased accumulation of ROS and of oxidative damaged products. Consequently, these mutants are more susceptible to UV-B-induced membrane damage and exhibits a higher levels of ascorbate peroxidase activity than wild type [11].

\section{Flavonoids and Melanins: The main Im- plications}

\section{Flavonoids}

Flavonoids are secondary metabolites synthesized via the phenylpropanoid pathway. They possess 15 carbon atoms and share a common $\mathrm{C}_{6}-\mathrm{C}_{3}-\mathrm{C}_{6}$ skeleton, with two benzene rings linked through a heterocyclic pyrane or a pyrone ring (Figure 1). According to the $C$ ring substitution pattern, six major subgroups of 
flavonoids can be identified: chalcones, flavones, flavonols, flavanones, anthocyanins and isoflavonoids. These compounds are bioactive across the plant kingdom with over 9,000 structural variants known [12]. Flavonoids act as scavengers of free radicals (e.g. ROS). Their antioxidant nature is defined mainly by the presence of a B-ring catechol group (dihydroxylated B-ring) capable of readily donating hydrogen (electron) to stabilize a radical species, in according to the following equation:

$$
\text { flavonoid }(\mathrm{OH})+\mathrm{R} \bullet>\text { flavonoid }(\mathrm{O} \bullet)+\mathrm{RH} \text {, }
$$

where $\mathrm{R} \bullet$ is a free radical and $\mathrm{O} \bullet$ is an oxygen free radical. Flavonols and anthocyanins are the best described antioxidant compounds and much information is available on their accumulation in plant exposed to UV radiations.

In maize, the exposure of plants to solar UV-B radiations led to the induction of all genes involved in flavonoid pathway: two flavonoid monooxigenases $(\mathrm{F} 3 \mathrm{H})$ and four glucosyltransferases (UFGT) [13]. UV-B radiations were found to induce the accumulate of specific flavonoids, such as kaempferol and quercetin glycosides, in Brassica napus [14] and in Betula pendula [15]. In barley, flavones are the major class of flavonoids [16] and saponarin accumulation in vacuoles is known to be an effective protecting against UV-B radiation [17].

An Arabidopsis group of mutants, collectively named transparent testa $(t t)$ mutants, are characterized by the reduction or the absence of pigments in the testa (seed coat), showing yellow or pale-brown seeds. Transparent testa mutants with impaired expression of two key enzymes of the flavonoid synthesis (chalcone synthase, CHS, and chalcone isomerase, CHI) are hypersensitive to UV-B radiation although, after UV-B treatment, they manifested only a modest growth retardation compared to wild-type. The unexpected

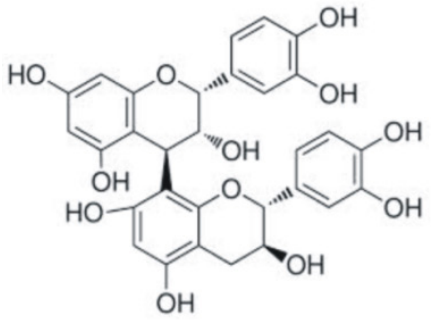

Procyanidin B2

Flavonoids tolerance of these mutants to UV-B may reflect the increased accumulation of other flavonoids [18]. Conversely, an Arabidopsis mutant (uvt1) with a constitutive elevated accumulation of anthocyanins due to high expression level of CHS gene are tolerant to UV-B radiation [19].

Beside a clear role in the UV-response, flavonoids exhibit also additional biological functions playing an important role in the interaction between plants and their environment [20]. Flavonoid biosynthetic genes are induced under several stress conditions and, accordingly, flavonoid levels increase during exposure to stresses, such as drought, low temperature, metal toxicity and pathogen attack [21-23]. The accumulation of ROS is the common feature of all these conditions. When wild type and flavonoid deficient Arabidopsis mutants $(t t 4, t t 5)$ were exposed to heavy metal either cadmium $(\mathrm{Cd})$ or zinc $(\mathrm{Zn})$, the seedling and root growth were considerably inhibited in $t t$ mutant lines [24] since flavonoids are effective in binding metals in acid soils because of competition with $\mathrm{H}+$ ions. Roots of maize plants exposed to $\mathrm{Al}$ exuded phenolics, and the degree of Al-resistance is dependent on the amount of flavonoids exuded [25].

Flavonoids act as signal molecules in plant-microorganism symbiosis [26]. Isoflavones are involved in nodule meristem formation activating nodulation (nod) genes and allowing the nitrogen fixation in legume species [27]. Flavonoids act also as phytoalexins, antimicrobial compounds synthesized at the site of infection in response to microbial attack. In rice and sorghum, they contribute to the resistance against Magnaporthe grisea and Colletotrichum spp. [28]. In hybrid poplar (Populus trichocarpa $\times$ P. deltoides) the response to infection by Melampsoralarici populina leaf rust and Marssonina brunnea, involves flavonoid accumulation $[29,30]$.

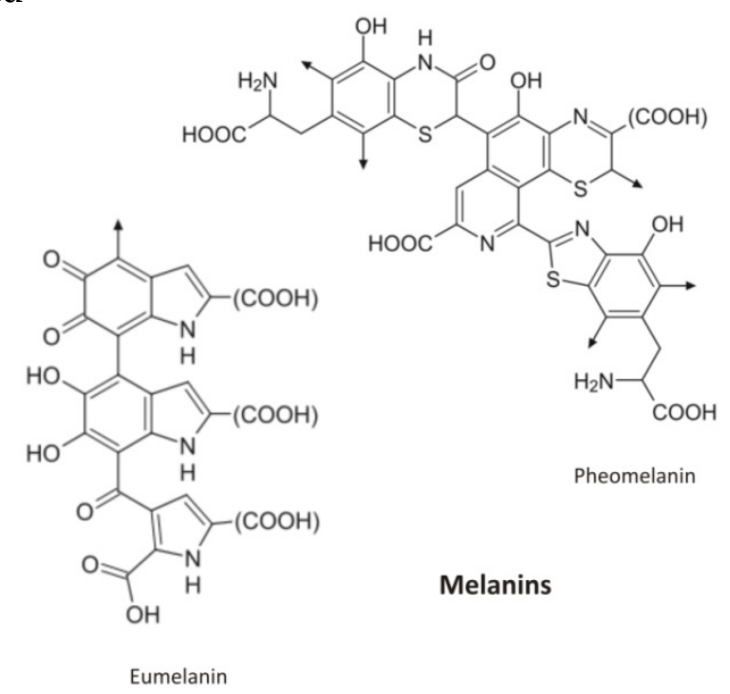

Figure I. Flavonoid (procyanidin B2) chemical structure and the building block of melanins (both eumelanin and pheomelanin) 
Proanthocyanidines (PAs), a class of flavonoid polymers resulting from the condensation of flavan-3-ol units, are associated with the brown seed coat or testa. They assist in the reinforcement of plant tissues, to the maintenance of seed dormancy as well as seed longevity in storage [31]. Transparent testa 12 (tt12) Arabidopsis mutant, that exhibit strong reduction in flavonoids deposition in seed endothelium, showed a shorter seed dormancy [32].

Anthocyanins are the molecules that confer flower pigmentation [33]. They are responsible, therefore, of pollinator attraction and consequently they play a role in plant reproduction. In fact, colour of flowers provides visual cues that lead pollinators to nectar-filled flowers and attract seed dispersers to ripened fruits [34]. In maize, at least two independent flavonoid biosynthetic pathways have been characterized. A first pathway leads to 3-hydroxy flavonoids such as anthocyanins purple pigment, whereas the other one produces 3-deoxy flavonoids such as the phlobaphene red pigment accumulated in kernel pericarp, silks and cob. Phlobaphenes are synthesized by the oxidation of colorless flavan-4-ol monomers or its polymers as shown in figure 2 .

\section{Melanins}

Melanins are heterogeneous metabolites composed by polyphenolic compounds. Melanin pigments are composed of different units connected through strong carbon-carbon bonds, which makes the systematic characterization of melanins very difficult.

Although the exact chemical structures of the melanins are still unknown due to the complex copolymerization and post polymerization modifications, four main classes can be recognized: eumelanin, pheomelanin (Figure 1), mixed melanins (a combination of the two), and neuromelanin [35].

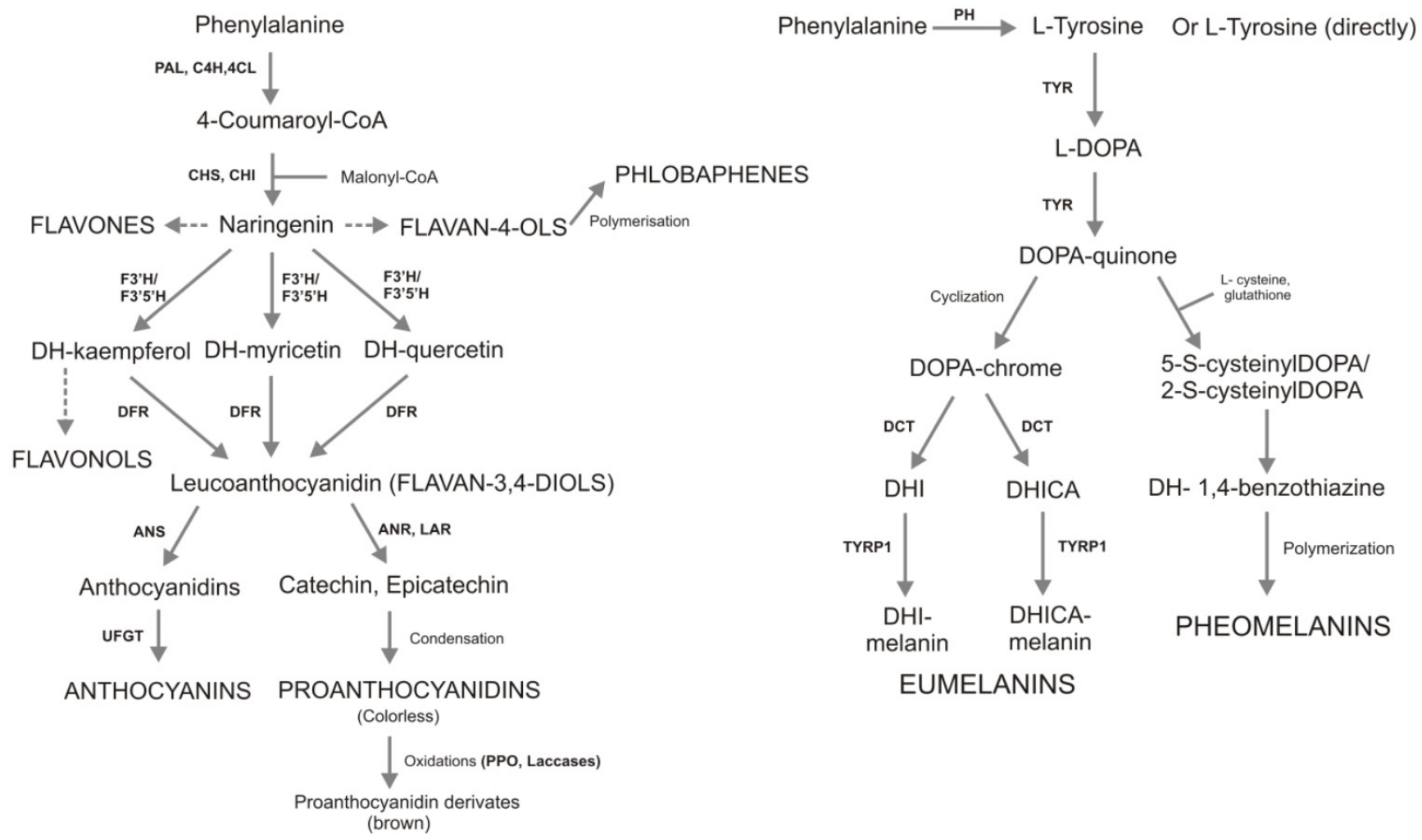

Figure 2. Synthetic metabolic pathways of flavonoids (on the left) and melanins (on the right). The synthesis of two antioxidant compounds starts from the same precursor (phenylalanine). Then, phenylalanine origins different molecules with the same biological functions. Flavonoid pathway are more branches compared to melanin synthesis, it has more enzymes and more than 9000 molecules are involved. Enzymes are reported with the following letter code: PAL, phenylalanine ammonia lyase; $\mathrm{C4H}$, cinnamate 4-hydroxylase; 4CL, coumarate:CoA ligase; CHS, chalcone synthase; $\mathrm{CHI}$, chalcone isomerase; $\mathrm{F} 3 \mathrm{H}$, flavanone 3b-hydroxylase; DFR, dihydroflavonol reductase; ANS, anthocyanidin synthase; ANR, anthocyanidin reduttase; LAR, leucoanthocyanidin reduttase; UFGT, UDP-glucose flavonol-0-glucosyl transferase; PH, Phenylalanine hydroxylase; TYR, tyrosinase; TRYPI, tyrosinase-related protein I; DCT, dopachrometautomerase; DOPA, L-3,4- dihydroxyphenylalanine; DHI, 5,6-dihydroxyindole; DHICA, indole-5,6-quinone carboxylic acid. In capital letters are reported theseven and the two main classes of flavonoids and melanins, respectively. 
Mammalian melanocytes produce two chemically distinct types of melanin pigments: black-brown eumelanin and yellow-reddish pheomelanin. Although they contain a common arrangement of repeating units linked by carbon-carbon bonds, melanin pigments differ each other's with respect to their chemical, structural, and physical properties [36]. Eumelanin consists of 5,6-Dihydroxyindole (DHI) and 5,6-dihydroxyindole-2-carboxylic acid (DHICA) units in a reduced or oxidized state, and some indole rings split to give pyrrole rings. On the other hand, pheomelanin consists mostly of benzothiazine units degraded, to some extent, to benzothiazole.

Thanks to semiquinone units the melanines act as redox pigment with both reducing and oxidizing capabilities towards oxygen radicals and other chemical redox systems [37]. Further, they also bind metals, whereupon it may switch to generate hydroxyl radicals, the most damaging ROS.

UV-induced melanogenesis is mediated via up-regulation of the MSH (Melanocyte Stimulating Hormone) receptor system [38]. Mice carrying an inactivating mutation at the $\mathrm{MSH}$ receptor $\left(\mathrm{Mc}^{\mathrm{e}} \mathrm{r} / \mathrm{e}\right)$ exposed to UV exhibited gross and histological evidence of sun damage: decrease in weight-gain, profound epidermal thickening and inflammation and scarring [39].

Melanins exhibit also secondary biological functions linked to their antioxidant properties and play an important role in the interaction between animals and their environment. Melanized Cryptococcus neoformans yeast cells are more resistant to silver nitrate, a compound highly toxic to bacteria and fungi, than non-melanized cells [40]. In fish melanins changes are often related to stress [41]. The main pigmentation controlling hormones, $\mathrm{MSH}$ and $\mathrm{MCH}$ (Melanin-Concentrating Hormone), also regulate the response to stress factors. The increased levels of $\mathrm{MCH}$ are known to influence the cortisol release and thus the stress response in a number of fish species, reducing the level of stress in the animals [42]. Spotted salmon (with higher amount of eumelanin) had significantly lower post-stress cortisol levels than those of their non-spotted conspecifics, furthermore spotted fish spent less time moving during acute stress when compared to non-spotted individuals [43].

Melanins have been shown to interfere with several host defense mechanisms. For instance, melanized fungus Cryptococcus neoformans cells manifest increased resistance to phagocytosis in vitro and in vivo [44] and in macrophage-like cell lines, the phagocytic index for melanized Paracoccidioides brasiliensis yeast cells was half that for the nonmelanized cells [45].

In many pathogen microorganisms melanins synthesis is associated with virulence towards both animal and plant hosts. Melanins are believed to contribute to microbial virulence by reducing a pathogen's susceptibility to host antimicrobial mechanisms and by influencing the host immune response to infection [46]. In microbes melanins are generally synthesized through various phenol-oxidases (such as tyrosinases, laccases, or catacholases) and/or the polyketide synthase pathways. Laccases are metalloproteins containing one to four copper binding sites with some homologies with tyrosinases [47], but, in contrast to tyrosinases, laccases are unable to convert tyrosine to 3,4-dihyroxyphenylalanine (L-DOPA). Chemical composition studies of melanins isolated from the Cryptococcus neoformans show that the C:N:O ratio is very similar to that of L-DOPA. This finding reveals that the synthesis of melanins in absence of tyrosinases results from polymerization of L-DOPA derivatives [48]. The structure of $C$. neoformans melanins remains elusive and very little is known about the evolution of laccases and tyrosinases. Both enzymes can be found in Eukaryota and in Prokaryota, which confirms their ancient evolutionary origin [47]. As reported before, laccases has been found also in plants [49]. In Arabidopsis, the transparent testa 10 (tt10) mutant is defective in the laccase-like enzyme TT10/LAC15, which is involved in the oxidative polymerization of flavonoids. $t+10$ mutant seeds show a clear $t t$ phenotype, which is generally associated with a reduction in the levels of brown, oxidized PAs [49]. Laccases are also considered a promoter of virulence in microbes. In C. neoformans laccase promotes virulence by inhibiting the oxidative burst in the phagosomal space of macrophages as a consequence of reducing $\mathrm{Fe}^{3+}$ to $\mathrm{Fe}^{2+}$ [50]. The marine bacterium Marinomonas mediterranea contains both tyrosinase and laccase, but only tyrosinase is required for the synthesis of the pigments [51]. The ability of pathogenic bacteria to produce melanin is originated from the evolutionary achievements of free-living bacteria. In some genera containing both free-living and parasitic strains (e.g. Vibrio sp.), there are both pyomelanogenetic (formed from either tyrosine or phenylalanine through the accumulation of homogentisic acid that has no identity with DOPA melanin) and eu/pheomelanogenetic pathways, sometimes simultaneously active in the same organism. Free-living strains of Vibrio cholerae are usually melanotic or pyomelanin producer [52]. Meanwhile, under stress they induce synthesis of eumelanin, suggesting how free-living bacteria need the presence of both laccases and tyrosinases to produce melanins and how the melanogenesis process depends from the environmental conditions.

Melanins also have a potential adaptive function 
in animal kingdom [53] where their patterns are widely associated to social interactions. The size of many melanin-based patches affects male dominance and an association between the presence of melanis, morphological and physiological traits and reproductive behavior has been found [54]. Darker wild vertebrates are generally more aggressive, sexually active and resistant to stress than lighter individuals. In the barn owl (Tyto alba) melanin-based coloration is associated with several behavioral, morphological and physiological characteristics linked to stress coping ability [55]. Its offspring mount stronger immune responses when their biological parents are darker even when they are raised by foster parents [56].

Melanins are also responsible for pigmentation of hairs, skins, cuticles, feathers and eyes. Their colour is mainly determined by the brown to black eumelanin and yellow to reddish-brown pheomelanin. In human, skin pigment contents are higher in regions of lower latitude and higher UV radiation levels. However, this connection may only be a recent human adaptation since early hominids may have possessed dark, dense, terminal body hairs. A closely related primate, the chimpanzee exhibits white or lightly pigmented epidermis [57]. Interestingly, chimpanzees have active melanocytes only in the epidermis of those areas directly exposed to UV radiation, face and friction surfaces [58].

Recent studies reveal that melanins are involved also in signal attraction and signal communication. The ability for the information transmission is mediated by colored traits, giving to melanins the role of signal interaction. Galván and Alonso-Alvarez [59] demonstrated this hypothesis in great tit (Parus major), a passerine bird that displays a well-known melanin-based signal, as black breast stripe. This trait serves as a badge of status in both sexes, larger stripes leading to higher success in agonistic interactions between conspecifics and more evident are the pigmentations more frequent is the mating between male and female.

Coloured traits are also associated to social dominance. In reptiles, melanins play an important role in thermoregulation. Darker individuals show lower skin reflectance, heat up more rapidly and can maintain their optimal body temperature better than lighter colored individuals at low temperatures. Consequently, darker individuals may have a higher fitness in colder and higher altitudinal regions than lighter coloured individuals [60]. Further, a correlation between the degree of aggressiveness and the eumelanic pigmentation of the carapace in Hermann's tortoises (Eurotestudo boettgeri) has also been reported [61]. A strong dark-coloured carapace is used to signal the degree of aggressiveness during male-male inter- actions and the individuals were more prudent in front of dark rather than pale conspecifics. The author hypothesize that dark tortoises may warm up more rapidly, as written before, allowing them to invest more energy in aggressive behavior.

\section{A Comparison between Flavonoid and Melanin Metabolic Pathways}

Figure 2 illustrates the biosynthetic pathway of flavonoids (A) and melanins (B), both starting from the L-phenylalanine. In plants, L-phenylalanine is deaminated to produce 4-coumaroyl-CoA that is condensed with malonyl-CoA to produce naringenin, the common precursor of all flavonoids. In animals, phenylalanine is hydroxylate to generate L-tirosine from which DOPA-quinone, the precursor of eumelanins and pheomelanins, is derived. Flavonoids and melanins are synthesized in the cytosol and transported conjugated to glutatione-S-transferase (GST) in specific cell organelles: vacuole and melanosomes, respectively. Flavonoid biosynthesis is characterized by a complex highly branched pathway involving many substrates and enzymes, and resulting in thousands of compounds. On the contrary melanins biosynthetic pathway involves three main compounds (L-3,4-dihydroxyphenylalanine-L-DOPA; DOPAquinone; DOPAchrome) and only three main enzymes: tyrosinase (TYR) controlling the synthesis of DOPA-quinone, tyrosinase-related protein 1 (TRYP1) and dopachrometautomerase (DCT; also known as tyrosinase-related protein 2, TYRP2) controlling the synthesis of eumelanins. The synthesis of pheomelanins takes place through spontaneous reactions (cyclization and polymerization).

While in melanins biosynthetic pathway, tyrosinase represents the only rate-limiting enzyme [62], in the case of flavonoids there are three main enzymes with a critical role in the regulation of the pathway: CHS (chalcone synthase), CHI (chalconeisomerase) and DFR (dihydroflavonol reductase) [63]. In Arabidopsis, three mutants, $t t 4, t t 5$ and $t t 3$, were generated in the main flavonoid controlling genes, $\mathrm{CHS}$, $C H I$ and DFR, respectively. Mutants $t t 4$ and $t t 5$ were totally deficient of flavonoids in all tissues, while $t+3$ mutant accumulated flavonols only in seeds, suggesting that these loci are required for the synthesis of all or major part of flavonoid products in Arabidopsis [64]. The over-expressed the CHI gene of Petunia hybrida in tomato plants led to an increased content of flavonol end products in the tomato fruit peel, highlighting that $\mathrm{CHI}$ enzyme activity could represent the sole rate-limiting step in this pathway [65].

Flavonoid end-products resembling the most animal melanins are condensed tannins (proanthocyanidins) because of their polymeric nature (fla- 
van-3-ol polymers). Their molecules (e.g. procyanidin B2, Figure 1) show similarity with melanins structure. Originally colorless, they exhibit a brownish colour upon oxidation by a polyphenol oxidase. Melanins, in the same way, are produced by the oxidation of the amino acid tyrosine, followed by polymerization.

\section{Flavonoids Effects on Animal Organisms}

Plants represent a primary source of nutrients for many animals and, therefore, animals ingest a relevant amount of flavonoids that, due to their similarity with melanins, might have implication onto the animal metabolism. Several flavonoids have estrogenic and progestational activity thanks to their core structure (diaryl ring), interfering with mammalian pigmentation [66]. Brzezinski and Debi [67] have suggested that phytoestrogens may represent natural selective estrogen receptor modulators (SERMs), remembering that human melanocytes express the functional estrogen receptor.

Due to their structure similarities with melanins, flavonoids are recognized as cofactors or substrates by the key enzyme of melanin biosynthesis (tyrosinase). Some flavonols, such as kaempferol and quercetin, possessing a 3-hydroxy-4-keto moiety similar to dihydroxyphenyl group in L-DOPA, may competitively inhibit tyrosinase activity due to their ability to chelate the copper in the active site [68]. These findings suggest a possible interfere of flavonoids with melanin biosynthesis and highlight, once again, a significant similarity in the properties of these two types of pigments, their ability to bind the same substrates and the same role in the two different kingdoms.

\section{Betalaines: A Possible link Between Melanins and Flavonoids}

Tyrosinase, the key enzyme of melanins biosynthesis, has also been found in several plant species capable to accumulate betalaine, a class of secondary metabolites including the red-violet betacyanins and the yellow betaxanthins [69]. Betalains accumulate only in some higher fungi and in Caryophyllaceae, considered an early and distinctive offshoot of Angiosperm. Besides betalains, Caryophyllaceae also produce flavonoids but not anthocyanins (the most antioxidant class of flavonoids). Noteworthy, betalains have never been found together with anthocyanins in the same plant species, suggesting that betalains and anthocyanins are evolution of mutually exclusive pathways [70]. Betalains differ from anthocyanins in composition and chemical structure, e.g. the former have a nitrogen group in their molecules whereas anthocyanins do not (Fig. 3a), although both are glycosilated and accumulated in response to light. The hydroxylation of tyrosine to DOPA and the further oxidation of DOPA, both catalyzed by tyrosinase, are considered the first steps in the betalain biosynthetic pathway. Then, DOPA undergoes an enzymatic extradiol cleavage leading via 4,5-seco-DOPA to betalamic acid, which likewise reacts spontaneously with cyclo- DOPA to form the betacyanin, or with various amino acids and amines to form the betaxanthin (Fig.3b). Harris et al. [71] were able to accumulate high levels of betalains (both betaxanthin and betacyanin) in non-betalain, anthocyanin-producing species (Antirrhinum, Arabidopsis and potato) by the over-expression of the gene coding for DOD (DOPA-4,5-dioxygenase). These results indicate the background presence of an enzyme able to convert L-DOPA to cyclo-DOPA, or dopaxanthin to betacyanin. Compared to flavonoids metabolism, betalains and melanins have a simpler biosynthetic pathway with the same rate limiting enzyme, suggesting that betalains are, at genetic and biochemical level, closer to melanin compounds. In contrast to melanins, betalains as well as anthocyanins are present as the glycosilated forms. All together these similarities might suggest that the betalains represent the transition pigments in plants, before the evolution of flavonoids and they could represent the joining pigments between flavonoids and melanins. With the evolution of Angiosperm, the antioxidant pigments have followed an evolutionary change, leading to the loss of betalains and the acquisition of flavonoids.

Several studies have demonstrated that most fungi produce melanins as antioxidant compounds. Betalains are accumulated in the higher fungi belonging to Basidiomycota class, conversely, no evidence for flavonoids compounds in fungi are known [72]. The molecular phylogenetic data demonstrate that animals and fungi belong to the same evolutionary group, named Opisthokonta [73-75] and this relationship could explain the affinity in UV protection compounds between animals and fungi.

\section{A Possible Common origin for UV Pro- tection Strategy in Plants and Animals}

The endosymbiontic theory identifies two specific groups of bacteria, a-proteobacteria and cyanobacteria, as the closest relatives of mitochondria and chloroplasts, respectively [76]. Many genes were either lost from plastids or transferred to the nucleus during the course of plant evolution [77]. It can be argued that melanins and flavonoids could have evolved from an ancient pigment produced in bacteria and after endosymbiosis some genes involved in pigment biosynthetic pathways have been integrated into animal and plant genomes. 


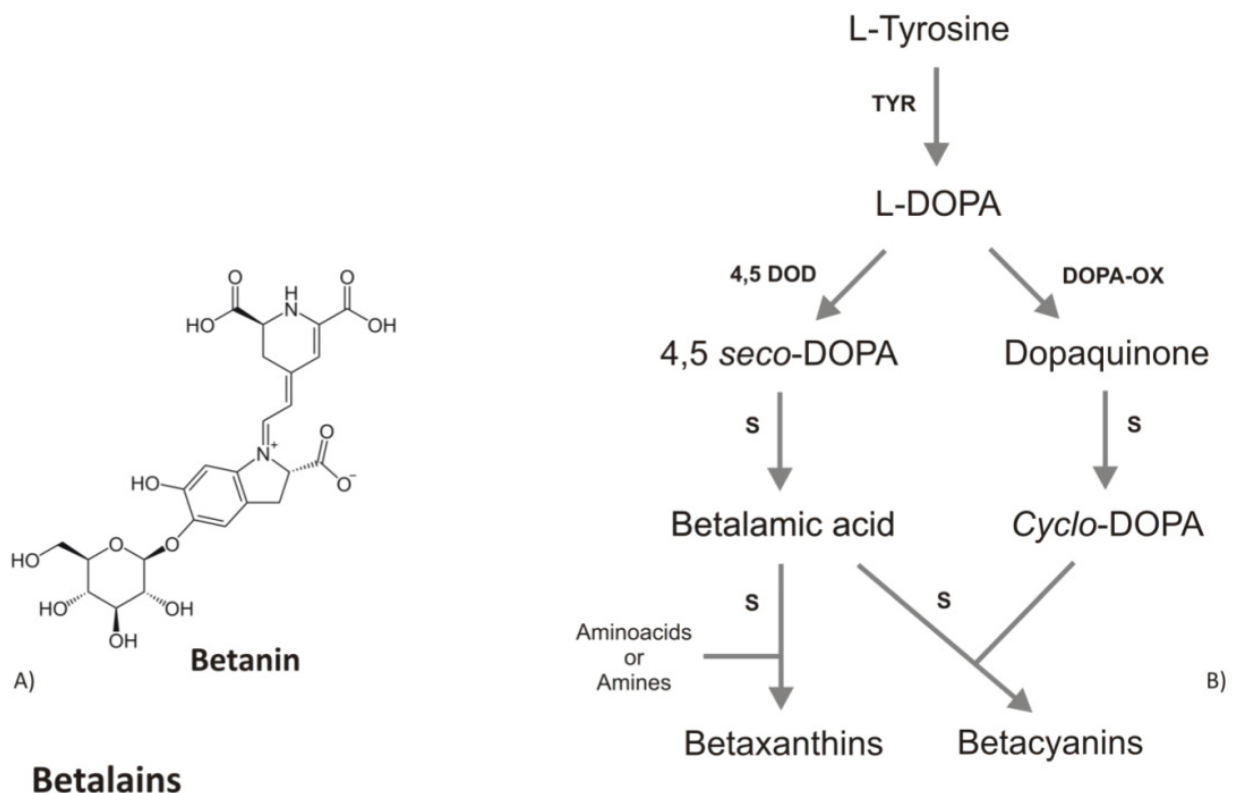

Figure 3. A) Chemical structure of betanin, the most studied betalain. B) Schematic biosynthetic pathway of betalains. Abbreviations are: TYR, tyrosinase; 4,5DOD, DOPA-4,5-dioxygenase; DOPA-OX, DOPA oxidase; $S$, spontaneous reaction.<smiles>COC1=C(NCC(=O)O)CC(O)(CO)CC1=NCC(=O)O</smiles>

Mycosporine-glycine

A)

MAA

Erythrose-4-phosphate

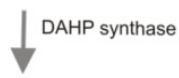

2-keto-3-deoxy-Darabinoheptulosinate-7phosphate

$\downarrow$ DHQ synthase

3-dehydroxyquinate

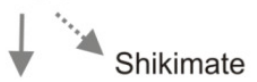

4-dehoxygadusol

- Glycine

Mycosporine-glycine

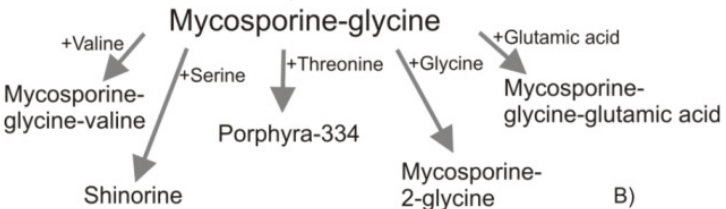

Figure 4. A) Mycosporine-2-glycine, one of the most abundant MAA in cyanobacteria. B) MAAs biosynthesis via shikimate pathway. PEP, phosphoenolpyruvate; DAHP, 3-deoxy-D-arabino-heptulosonate- 7-phosphate; DHQ, 3-dehydroquinate.

Cyanobacteria, the ancient organism involved in endosymbiosis with plant kingdom, produces mycosporine amino acids (MAAs), small secondary metabolites able to absorb UV radiations [78] and to confer resistance to several abiotic stresses [79]. MAAs are known from taxonomically diverse organisms, including many marine groups such as heterotrophic bacteria [80] and micro/macroalgae. Many animals such as arthropods, mollusks, fishes, cnidarians, and protozoans also accumulate MAAs to protect themselves from UV radiation [81].

All known MAAs are composed by a central cyclohexenone or cyclohexenimine ring with a wide variety of substitutions (Figure 4a). Recent studies of comparative genomics and bioinformatics reveal the presence of cyanobacteria MAA genes in other organisms such as dinoflagellates [82] and metazoan [83]. The authors hypothesized that the homologues of MAAs core biosynthesis genes has been transferred from cyanobacteria to dinoflagellates or metazoan by lateral gene transfer events (via a prokaryote-toeukaryote lateral/horizontal gene transfer) during the evolution, highlighting the theory of horizontal gene transfer (HGT) among bacteria, plants and animals. Several works report the implication of HGT in land colonization and their subsequent evolution [-84], 
conferring to the recipient organisms novel metabolic capabilities, e.g., it is known the crucial role of HGT from soil bacteria in the phenylpropanoids emergence [85]. Mycosporines and MAAs are synthesized in prokaryote and marine organisms (bacteria, cyanobacteria, phyto-plankton, macroalgae) but not in animals. The accumulation of MAAs in animals sometimes reported, is due to their algal diet [86] or to translocation from algal symbionts or associated bacteria [87]. The MAAs in eukaryotic algae are thought to have been passed from cyanobacteria in the plastid line. Portwich and Garcia-Pichel [88] demonstrated that the central ring of the MAAs in cyanobacteria derives from the shikimate pathway as in eukaryote and it may be speculated that early land plants initially were dependent on MAAs instead of flavonoids, as protectants from UV radiation [89].

The scientific information reported above suggests that cyanobacteria possess a precursor of the UV protecting mechanisms found in plants and animals. It can be hypothesized that before the land colonization, when all organisms lived in aquatic environment, an HGT took place. Bacteria, in particular cya-

\section{Organism Antioxidant molecule}

\section{Cyanobacteria/} Microalgae

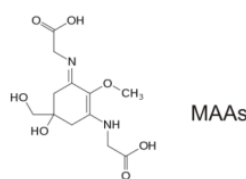

Caryophyllaceae/ Higher Fungi

Animals/Fungi

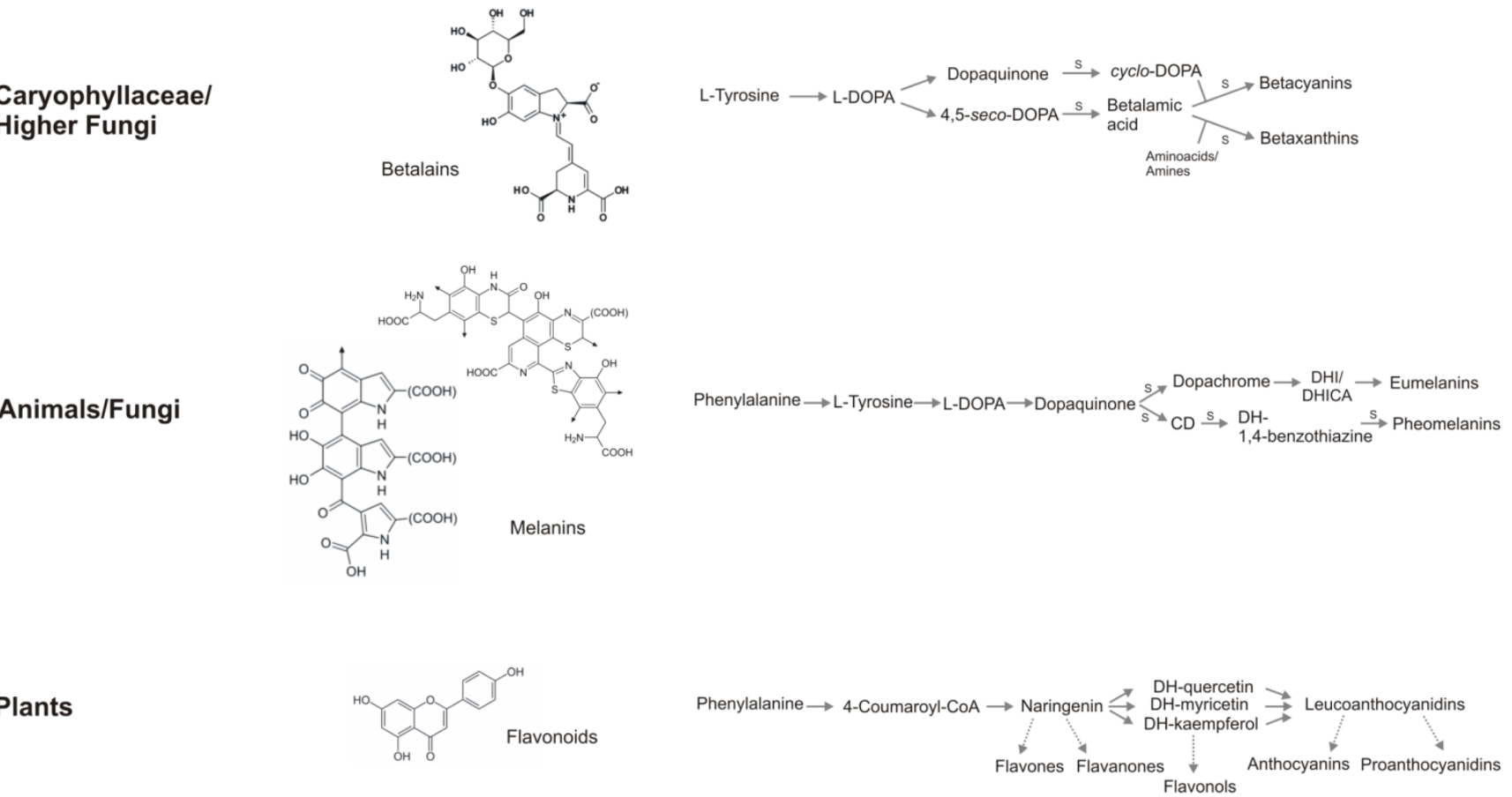

nobacteria, transferred to plants and animals the genes able to confer UV protection. With the emersion from water and the exposure to a higher UV radiation level the living organisms needed to produce more effective antioxidant molecules. Therefore, starting from the genes and pathways controlling MAAs biosynthesis, they evolved new protective compounds such as betalains, melanins and flavonoids. The betalains, present in higher fungi (Basidiomiceteae) and Caryophyllaceae, can be considered the first type of complex antioxidant compound evolved after water emersion. Along with the evolution, plant species became characterized by flavonoids, while melanins were evolved in animals. Fungi, closer to animals than plants, accumulate only melanins, (with the exception of Basidiomicetae) and, at least at biochemical level, betalains can also be considered the direct precursor of melanins.

In figure 5, a summary of the antioxidant strategies described in this review is reported: the chemical structure of molecules involved in cell protection and the skeleton of the biosynthetic pathway of MAAs, betalains, melanins and flavonoids are compared.

\section{Antioxidant metabolic pathway}

Figure 5. A summary of the antioxidant strategies in vivant. Flavonoid core, even if possess aromatic rings as the other antioxidant molecules, does not share other structural characteristics with MAAs, betalains, and melanins and does not contain nitrogen. Metabolic pathway of betalains and melanins are simple, several reaction are spontaneous, whereas flavonoid biosynthesis involves many reactions mediated by enzymes and several branches, that lead to several classes of flavonoids. It is interesting how the complexity of chemical molecules and the metabolic pathway follow an inverse proportion: melanins possess the most complex structure and the simplest biosynthetic pathway, the contrary occurs in the flavonoid metabolism. S: spontaneous chemical reaction. 


\section{Functional Analogies or Evolutionary Relationship?}

Available literature data suggest that highly effective protective strategies are conserved at biochemical level through different kingdoms. Melanin and flavonoid metabolic pathways depict a complex network involving a range of biological stimuli, biochemical responses and genetic interactions. Noteworthy, the antioxidant mechanisms are a perfect across kingdom example of a very similar response to a common evolutive pressure. Exposure of all living organisms to UV radiations has resulted in the evolution of very similar defensive strategies and molecules and these compounds; beside the common function (UV protection) also have a number of secondary roles related to the interactions among individuals and species.

In this work we have reported on a number of biochemical and functional analogies between UV-protection mechanisms in plants and animals, whether these analogies also reflect an evolutionary relationship is still under debate. Certainly, the response to UV in all organisms is the result of the same evolutive pressure, and this gives a reason for the analogies between the two strategies undertaken in plants and animals. The finding that some intermediate taxonomic groups contains betalains, the close relationship between the pathways of betalains and melanins (tyrosinase enzyme, L-DOPA intermediate), they presence in phylogenetic related organisms (higher fungi and animals) and the fact that betalains and anthocyanin are mutually exclusive might suggest that the biosynthetic pathways of betalains and melanins are parts of the same evolutionary process. On the other side, it is doubtful if an evolutionary relationship exists between MAAs and flavonoids or MAAs and melanins. At present, few enzymes involved in these pathways have been isolated [90] and some details of the metabolic processes are not well known. The genes coding for Phenylalanine Ammonia Lyase (PAL), the first enzyme involved in flavonoid biosynthesis, have been identified in the genomes of several prokaryotes, and an in vitro characterization of two prokaryotic PALs confirmed that they share many characteristics with eukaryotic PAL, including the catalytic activities [91]. Despite these results, it should be noticed that melanin and flavonoid pathways, do not share any enzymes or intermediates and that the flavonoid pathway is much more complex and branched than melanin metabolism. These large differences suggest that flavonoids and melanines pathways most likely represent an example of functional analogies.

\section{Competing Interests}

The authors have declared that no competing interest exists.

\section{References}

1. Rozema J, Björn LO, Bornman JF, Gaberscik A, Häder DP, Trost T, et al. The role of UV-B radiation in aquatic and terrestrial ecosystems--an experimental and functional analysis of the evolution of UV-absorbing compounds. J Photochem Photobiol B. 2002; 66:2-12.

2. Hideg E, Jansen MAK, Strid A. UV-B exposure, ROS, and stress: inseparable companions or loosely linked associates? Trends Plant Sci. 2012;:1-9.

3. Marrot L, Meunier JR. Skin DNA photodamage and its biological consequences. J Am Acad Dermatol. 2008; 58:S139-S148.

4. Bose B, Agarwal S, Chatterjee SN. UV-A induced lipid peroxidation in liposomal membrane. Radiat Environ Biophys. 1989; 28:59-65.

5. Kaidbey KH, Agin PP, Sayre RM, Kligman AM. Photoprotection by melanin a comparison of black and Caucasian skin. J Am Acad Dermatol. 1979; 1:249-260.

6. Halder RM, Bridgeman-Shah S. Skin cancer in African Americans. Cancer. 1995; 75 (Suppl 2):667-673

7. Szabo GS, Gerald AB, Pathak MA, Fitzpatrick TB. Racial differences in the fate of melanosomes in human epidermis. Nature. 1969; 222:1081-1082.

8. Kobayashi M, Aita N, Hayashi S, Okada K, Ohta T, Hirose S. DNA supercoiling factor localizes to puffs on polytene chromosomes in Drosophila melanogaster. Mol Cell Biol. 1998; 18(11):6737-6744.

9. Bustamante C, Keller D, Yang G. Scanning force microscopy of nucleic acids and nucleoprotein assemblies. Curr Opin Struct Biol. 1993; 3:363-372.

10. Mazza CA, Boccalandro HE, Giordano CV, Battista D, Scopel AL, Ballaré CL. Functional significance and induction by solar radiation of ultraviolet-absorbing sunscreens in field-grown soybean crops. Plant Physiol 2000; 122:117-125.

11. Landry LG, Chapple CCS, Last RL. Arabidopsis mutants lacking phenolic sunscreens exhibit enhanced ultraviolet-B injury and oxidative damage. Plant Physiol. 1995; 109:1159-1166.

12. Williams RJ, Spencer JPE, Rice-Evans C. Flavonoids: antioxidants or signalling molecules? Free Radic Biol Med. 2004; 36:838-849.

13. Casati P, Campi M, Morrow DJ, Fernandes JF, Walbot V. Transcriptomic, proteomic and metabolomic analysis of UV-B signaling in maize. BMC Genomics. 2011; 12:321.

14. Wilson KE, Thompson JE, Huner NP, Greenberg BM. Effects of ultraviolet-A exposure on ultraviolet-B-induced accumulation of specific flavonoids in Brassica napus. Photochem Photobiol. 2001; 73(6):678-84.

15. Kotilainen $T$, Venäläinen $T$, Tegelberg $R$, Lindfors A, Julkunen-Tiitto $R$, Sutinen S, et al. Assessment of biological spectral weighting functions for phenolic metabolites and growth responses in silver birch seedlings. Photochem Photobiol. 2009; 85: 1346-1355.

16. Ferreres F, Andrade PB, Valentao P, Gil-Izquierdo A. Further knowledge on barley (Hordeum vulgare L) leaves O-glycosyl-Cglycosyl flavones by liquid chromatography-UV diode-array detection- electrospray ionisation mass spectrometry. J Chromatogr A. 2008; 1182: 56-64.

17. Schmitz-Hoerner R, Weissenböck G. Contribution of phenolic compounds to the UV-B screening capacity of developing barley primary leaves in relation to DNA damage and repair under elevated UV-B levels. Phytochemistry. 2003; 64: $243-255$

18. Booij-James IS, Dube SK, Jansen MA, Edelman M, Mattoo AK. Ultraviolet-B radiation impacts light-mediated turnover of the photosystem II reaction center heterodimer in Arabidopsis mutants altered in phenolic metabolism. Plant Physiol. 2000; 124:1275-1284.

19. Bieza K, Lois R. An Arabidopsis mutant tolerant to lethal ultraviolet-B levels shows constitutively elevated accumulation of flavonoids and other phenolics. Plant Physiol. 2001; 126:1105-1115.

20. Pourcel L, Routaboul JM, Cheynier V, Lepiniec L, Debeaujon I. Flavonoid oxidation in plants: from biochemical properties to physiological functions. Trends Plant Sci. 2007; 12:29-36.

21. Winkel-Shirley B. Biosynthesis of flavonoids and effects of stress. Curr Opin Plant Biol. 2002; 5:218-223.

22. Løvdal T, Olsen KM, Slimestad R, Verheul M, Lillo C. Synergetic effects of nitrogen depletion, temperature, and light on the content of phenolic compounds and gene expression in leaves of tomato. Phytochemistry. 2010; 71:605-613.

23. Oh M, Carey EE. Regulated water deficits improve phytochemical concentration in lettuce. J Am Soc Hort Sci. 2010; 135:223-229.

24. Keilig K, Ludwig-Müller J. Effect of flavonoids on heavy metal tolerance in Arabidopsis thaliana seedlings. Bot Stud. 2009; 50:311-318.

25. Kidd PS, Llugany M, Poschenrieder C, Gunsé B, Barceló J. The role of root exudates in aluminium resistance and silicon-induced amelioration of aluminium toxicity in three variety of maize (Zea mays L). J Exp Bot. 2001; 52:1339-1352.

26. Dixon RA, Steele CL. A gold mine for metabolic engineering. Trends Plant Sc. 1999;: 1380-1385. 
27. Hassan S, Mathesius U. The role of flavonoids in root-rhizospheresignalling: opportunities and challenges for improving plant-microbe interactions. J Exp Bot. 2012; 63:3429-3444.

28. Ibraheem F, Gaffoor I, Chopra S. Flavonoid phytoalexin-dependent resistance to anthracnose leaf blight requires a functional yellow seed1 in Sorghum bicolor. Genetics. 2010; 184:915-926.

29. Miranda M, Ralph SG, Mellway R, White R, Heath MC, Bohlmann J, et al. The transcriptional response of hybrid poplar (Populus trichocarpa $\times$ P. deltoides) to infection by Melampsora medusae leaf rust involves induction of flavonoid pathway genes leading to the accumulation of proanthocyanidins. Mol Plant Microbe Interact. 2007; 20:816-831.

30. Yuan L, Wang L, Han Z, Jiang Y, Zhao L, Liu H, et al. Molecular cloning and characterization of PtrLAR3, a gene encoding leucoanthocyanidin reductase from Populus trichocarpa, and its constitutive expression enhances fungal resistance in transgenic plants. J Exp Bot. 2012; 63:2513-2524.

31. Lepiniec L, Debeaujon I, Routaboul JM, Baudry A, Pourcel L, Nesi N, et al. Genetics and biochemistry of seed flavonoids. Ann Rev Plant Biol. 2006; 57:405-430.

32. Debeaujon I, Peeters AJ, Léon-Kloosterziel KM, Koornneef M. The TRANSPARENT TESTA12 gene of Arabidopsis encodes a multidrug secondary transporter-like protein required for flavonoid sequestration in vacuoles of the seed coat endothelium. Plant Cell. 2001; 13:853-871.

33. Winkel-Shirley B. Flavonoid Biosynthesis A Colorful Model for Genetics, Biochemistry, Cell Biology, and Biotechnology. Plant Physiol. 2001; 126:485-493.

34. Kennedy JA, Matthews MA, Waterhouse AL. Changes in grape seed polyphenols during fruit ripening. Phytochemistry. 2000; 55:77-85.

35. Watt R, Bothma JP, Meredith P. The supramolecular structure of melanin. Soft Matter. 2009; 5:3754-3760.

36. Ito S. The IFPCS presidential lecture: a chemist's view of melanogenesis. Pigment Cell Res. 2003:: 230-236.

37. Prota G. The chemistry of melanins and melanogenesis. Fortsch Chem Organ Natur. 1995; 64:93-148

38. Pawelek J, Chakraborty A, Osber MP, Orlow SJ, Min KK, Rosenzweig KE, et al. Molecular cascades in UV induced melanogenesis: a central role for melanotropins? Pigment Cell Res. 1992; 5:34-356.

39. D'Orazio JA, Nobuhisa T, Cui R, Arya M, Spry M, Wakamatsu K, et al. Topical drug rescue strategy and skin protection based on the role of Mc1r in UV-induced tanning. Nature. 2006; 21:340-344.

40. Garcia-Rivera J, Casadevall A. Melanization of Cryptococcus neoformans reduces its susceptibility to the antimicrobial effects of silver nitrate. Med Mycol. 2001; 39:353-357.

41. Arends RJ, Rotllant J, Metz JR, Mancera JM, Wendelaar-Bonga SE, Flik G. alpha-MSH acetylation in the pituitary gland of the sea bream (Sparusaurata L) in response to different backgrounds, confinement and air exposure. J Endocrinol. 2000; 166:427- 435 .

42. WendelaarBonga SE. The stress response in fish. Physiol Rev. 1997; 77:591625.

43. Kittilsen S, Schjolden J, Beitnes-Johansen I, Shaw JC, Pottinger TG, Sørensen C, et al. Melanin-based skin spots reflect stress responsiveness in salmonid fish. Horm Behav. 2009; 56:292-298.

44. Mednick AJ, Nosanchuk JD, Casadevall A. Melanization of Cryptococcus neoformans affects lung inflammatory responses during cryptococcal infection. Infect Immun. 2012; 73:2012-2019.

45. da Silva MB, Marques AF, Nosanchuk JD, Casadevall A Travassos LR, Taborda CP. Melanin in the dimorphic fungal pathogen Paracoccidioides brasiliensis: effects on phagocytosis, intracellular resistance and drug susceptibility. Microbes Infect. 2006; 8:197-205.

46. Nosanchuk JD, Casadevall A. The contribution of melanin to microbial pathogenesis. Cellular Microbiology. 2003; 5:203-223.

47. Valderrama B, Oliver P, Medrano-Soto A, Vazquez-Duhalt R. Evolutionary and structural diversity of $\backslash$ fungal laccases. Antonie van Leeuwenhoek. 2003; 84:289-299.

48. Wheeler MH, Bell AA. Melanins and their importance in pathogenic fungi. Curr Topics Med Mycol. 1988; 2:338-387.

49. Pourcel L, Routaboul JM, Kerhoas L, Caboche M, Lepiniec L, Debeaujon I. TRANSPARENT TESTA10 encodes a laccase-like enzyme involved in oxidative polymerization of flavonoids in Arabidopsis seed coat. Plant Cell. 2005, 17(11):2966-80

50. Liu L, Tewari RP, Williamson PR. Laccase protects Cryptococcus neoformans from antifungal activity of alveolar macrophages. Infect Immunol. 1999; 67:6034-6039.

51. López-Serrano D, Sanchez-Amat A, Solano F. Cloning and molecular characterization of a SDS-activated tyrosinase from Marinomonas mediterranea. Pigment Cell Res. 2002; 15:104-111.

52. Kotob S, Coon SI, Quintero EJ, Weiner RM. Homogentisic acid is the primary precursor of melanin synthesis in Vibrio cholerae, a Hyphomonas strain and Shewanel la colwelliana. Appl Environ Microbiol. 1995; 61: 1620-162.

53. McGraw KJ. Mechanics of melanin-based coloration. In: Bird Coloration, Vol I, eds. GE Hill \& K McGraw, Harvard University Press, Harvard. 2006:243- 294.

54. Roulin A. The evolution, maintenance and adaptive function of genetic colour polymorphism in birds. Biol Rev Camb Philos Soc. 2004; 79:815-848.

55. Almasi B, Roulin A, Jenni-Eiermann S, Jenni L. Parental investment and its sensitivity to corticosterone is linked to melanin-based coloration in barn owls. Horm Behav. 2008; 54:217-223.
56. Roulin A, Jungi TW, Pfister $\mathrm{H}$, Dijkstra C. Female barn owls (Tyto alba) advertise good genes. Proc Biol Sci. 2000; 267:937-941.

57. Post PW, Daniels F Jr, Binford RT Jr. Cold injury and the evolution of "white" skin. Hum Biol. 1975; 47:65-80.

58. Jablonski NG, Chaplin G. The evolution of human skin coloration. J Hum Evol. 2000; 39:57-106.

59. Galván I, Alonso-Alvarez C. The expression of melanin-based plumage is separately modulated by exogenous oxidative stress and a melanocortin. Proc Biol Sci. 2009; 276:3089-3097.

60. Clusella Trullas S, van Wyk JH, Spotila JR. Thermal melanism in ectotherms. J Therm Biol. 2007; 32:235-245.

61. Mafli A, Wakamatsu K, Roulin A. Melanin-based coloration predicts aggressiveness and boldness in captive eastern Hermann tortoises. Anim Behav. 2011; 81:859-863.

62. Chang TS. Natural Melanogenesis Inhibitors Acting Through the Down-Regulation of Tyrosinase Activity. Materials. 2012; 5:1661-1685.

63. Dong X, Braun EL, Grotewold E. Functional Conservation of Plant Secondary Metabolic Enzymes Revealed by Complementation of Arabidopsis Flavonoid Mutants with Maize Genes. Plant Physiol. 2001; 127:46-57.

64. Shirley BW, Kubasek WL, Storz GB, Ruggemann E, Koornneef M, Ausubel FM, et al. Analysis of Arabidopsis mutants deficient in flavonoid biosynthesis. Plant J. 1995; 8(5):659-671

65. Muir SR, Collins GJ, Robinson S, Hughes S, Bovy A, Ric De Vos CH, et al. Overexpression of petunia chalcone isomerase in tomato results in fruit containing increased levels of flavonols. Nat Biotechnol. 2001; 19:470-474.

66. Rosenberg Zand RS, Jenkins DJA, Diamandi EP. Steroid hormone activity of flavonoids and related compounds. Breast Cancer Res Treat. 2000; 62:35-49.

67. Brzezinski A, Debi A. Phytoestrogens: the 'natural' selective estrogen receptor modulators? Eur J Obstet Gynecol Reprod Biol. 1999; 85:47-51.

68. Kim YJ, Uyama H. Tyrosinase inhibitors from natural and synthetic sources: structure inhibition mechanism and perspective for the future. Cell Mol Life Sci.2005; 62:1707-1723.

69. van Gelder CWG, Flurkey WH, Wichers HJ. Sequence and structural features of plant and fungal tyrosinases. Phytochemistry. 1997; 45:1309-1323.

70. Stafford HA. Anthocyanins and betalains: evolution of the mutually exclusive pathways. Plant Sci. 1994; 101:91-98

71. Harris NN, Javellana J, Davies KM, Lewis DH, Jameson PE, Deroles SC. Betalain production is possible in anthocyanin-producing plant species given the presence of DOPA-dioxygenase and L-DOPA. BMC Plant Biol. 2012; 12:34.

72. Eisenman HC, Casadevall A. Synthesis and assembly of fungal melanin. Appl Microbiol Biotechnol. 2012; 93: 931-940.

73. Cavalier-Smith T. A revised six-kingdom system of life. Biol Rev Camb Philos Soc. 1998; 73:203-266.

74. Steenkamp ET, Wright J, Baldauf SL. The protistan origins of animals and fungi. Mol Biol Evol. 2006; 23:93-106.

75. Torruella G, Derelle R, Paps J, Lang BF, Roger AJ, Shalchian-Tabrizi K, et al. Phylogenetic Relationships within the Opisthokonta Based on Phylogenomic Analyses of Conserved Single-Copy Protein Domains. Mol Biol Evol. 2012; 29:531-544.

76. Gray MW, Spencer DF. Organellar evolution. In Evolution of Microbial Life, ed DM Roberts P, Sharp G Alderson, M Collins. Cambridge: Cambridge Univ Press 1996; 109-126.

77. Martin W, Rujan T, Richly E, Hansen A, Cornelsen S, Lins T, et al. Evolutionary analysis of Arabidopsis, cyanobacterial, and chloroplast genomes reveals plastid phylogeny and thousands of cyanobacterial genes in the nucleus. Proc Natl Acad Sci U S A. 2002; 99:12246-12251.

78. Ehling-Schulz M, Scherer S. UV protection in cyanobacteria. Eur J Phycol. 1999; 34(4):329-338.

79. Conde FR, Churio MS, Previtali CM. The photoprotector mechanism of mycosporine-like amino acids excited-state properties and photostability of porphyra-334 in aqueous solution. J Photochem Photobiol B. 2000; 56:139-144.

80. Arai T, Nishijima M, Adachi $\mathrm{K}$, Sano $\mathrm{H}$. Isolation and structure of a UV absorbing substance from the marine bacterium Micrococcus sp AK-334. Marine Biotechnology Institute Tokyo. 1992;: 88-94.

81. Sinha RP, Singh SP, Häder DP. Database on mycosporines and mycosporine-like amino acids (MAAs) in fungi, cyanobacteria, macroalgae, phytoplankton and animals. J Photochem Photobiol B. 2007; 89: 29-35.

82. Singh SP, Häder DP, Sinha RP. Bioinformatics evidence for the transfer of mycosporine-like amino acid core (4-deoxygadusol) synthesizing gene from cyanobacteria to dinoflagellates and an attempt to mutate the same gene (YP_324358) in Anabaena variabilis PCC 7937. Gene. 2012; 500:155-163.

83. Starcevic A, Akthar S, Dunlap WC, Shick JM, Hranueli D, Cullum J, et al. Enzymes of the shikimic acid pathway encoded in the genome of a basal metazoan, Nematostella vectensis, have microbial origins. Proc Natl Acad Sci U S A. 2008; 105:2533-2537.

84. Yue J, Hu X, Huang J. Horizontal gene transfer in the innovation and adaptation of land plants. Plant Signal Behav. 2013; 8: e24130.

85. Emiliani G, Fondi M, Fani R, Gribaldo SA. Horizontal gene transfer at the origin of phenylpropanoid metabolism: a key adaptation of plants to land. Biol Direct. 2009:* 4-7.

86. Newman SJ, Dunlap WC, Nicol S, Ritz D. Antarctic krill (Euphausia superba) acquire a UV-absorbing mycosporine-like amino acid from dietary algae. J Exp Mar Biol Ecol. 2000; 255:93-110.

87. Shick JM, Romaine-Lioud S, Ferrier-Pages C, Gattuso JP. Ultraviolet-B radiation stimulates shikimate pathway dependent accumulation of mycospor- 
ine-like amino acids in the coral Stylophora pistillata despite decreases in its population of symbiotic dinoflagellates. Limnol Oceanogr. 1999; 44:1667-1682.

88. Portwich A, Garcia-Pichel F. Biosynthetic pathway of mycosporines (mycosporine-like amino acids) in the cyanobacterium Chlorogloeopsis sp strain PCC 6912. Phycologia. 2003; 42:384-392.

89. McKenzie RL, Bjorn LO, Bais A, Ilyasd M. Changes in biologically active ultraviolet radiation reaching the Earth's surface. Photochem Photobiol Sci. 2003;2:5-15.

90. Shick JM. The continuity and intensity of ultraviolet irradiation affect the kinetics of biosynthesis, accumulation, and conversion of mycosporine-like amino acids (MAAs) in the coral Stylophora pistillata. Limnol Oceanogr. 2004; 49(2):442-458

91. Moffitt MC, Louie GV, Bowman ME, Pence J, Joseph P, Moore BS. Discovery of Two Cyanobacterial Phenylalanine Ammonia Lyases: Kinetic and Structural Characterization. Biochemistry. 2008; 46:1004-1012. 